\title{
Afrontamiento y adaptación de cuidadores de niños y niñas con cáncer
}

\author{
Coping and adaptation of caregivers of children with cancer
}

Sandra Milena Campiño-Valderrama', Paula Andrea Duque ${ }^{2}$

1 Enfermera, Especialista en Docencia Universitaria, Docente Auxiliar Universidad Católica de Manizales. e-mail: scampino@ucm.edu.co

2 Enfermera, Magister en Educación y Desarrollo Humano, Especialista en Administración de la Salud, Docente Asociada Universidad Católica de Manizales. e-mail: paduque@ucm.edu.co

\begin{abstract}
Resumen
Objetivo: Identificar la capacidad de afrontamiento y adaptación de cuidadores de niños y niñas con cáncer teniendo como referente el modelo de adaptación de Callista Roy. Materiales y métodos: Estudio con enfoque cuantitativo de tipo descriptivo y de corte transversal, cuya muestra estuvo constituida por 23 cuidadoras principales de niños y niñas con cáncer que se encontraban hospitalizados o asistían a quimioterapia ambulatoria. Para la recolección de la información se utilizó la Escala de Medición del Proceso de Afrontamiento y Adaptación de Callista Roy versión abreviada en español. Resultados: El 100\% de los cuidadores principales eran madres y abuelas. El 56,5\% de ellas se presentaba en un afrontamiento alto y el 43,5\% afrontamiento medio. Las cuidadoras que demostraron afrontamiento alto se ubican en el grupo de las que se encontraban solteras y casadas, pertenecientes al estrato uno, con nivel de escolaridad bachiller completo y llevaban menos de un año a cargo del cuidado. Los resultados por factores de afrontamiento demuestran reacciones, comportamientos y estrategias utilizadas para hacer frente a los problemas difíciles mediante un afrontamiento activo. Conclusión: Las características del cuidador y las estrategias utilizadas para conseguir respuestas adaptativas, deben ser aprovechadas por enfermería para promover un modelo de cuidado integral donde se aborde no solamente al niño o niña, sino también a su familia y entorno, con el fin de posibilitar un afrontamiento integrado.
\end{abstract}

Palabras clave: Cuidadores; estrategias de afrontamiento; adaptación psicológica. (Fuente: DeCS, Bireme).

\begin{abstract}
Objective: To identify the coping capacity and adaptation of caregivers of children with cancer taking as reference the adaptation model of Callista Roy. Materials and methods: A descriptive and cross-sectional study with a quantitative approach, whose sample consisted of 23 primary caregivers of children with cancer, who were hospitalized or attending outpatient chemotherapy. The Measurement Scale of Coping and Adaptation Process of Callista Roy abridged version in Spanish was used for data collection. Results: $100 \%$ of primary caregivers were mothers and grandmothers. 56.5\% of them presented high coping and $43.5 \%$ an average one. Carers who demonstrated high coping were single and married, belonging to strata 1 , with full high school education level and were in charge of care for less than a year. The results by factors of coping demonstrate reactions, behaviors and strategies used to address difficult problems through an active coping. Conclusion: The characteristics of the caregiver and the strategies used to achieve
\end{abstract}


adaptive responses should be used by nursing to promote a model of integrated care where not only the children but also their family and environment is addressed in order to enable an integrated coping.

Keywords: Caregivers; coping strategies; psychological adaptation. (Source: DeCS, Bireme).

\section{Introducción}

En la actualidad el cáncer es catalogado como una enfermedad crónica no transmisible (ECNT) que requiere ser abordada desde una perspectiva interdisciplinar, con el fin de lograr un mejor afrontamiento y adaptación de todos los hechos que guardan relación con esta enfermedad. En este sentido, es importante tener en cuenta, que el cáncer en los niños(as) presenta un comportamiento diferente al de los adultos en cuanto al tipo de enfermedad, evolución clínica, tratamiento y sobrevida; además, las probabilidades de curación de los niños(as) son $30 \%$ más altas que las de adultos de manera global, pudiendo llegar a un $85 \%$ de curación ${ }^{1}$.

A nivel mundial aproximadamente 200.000 niños(as) y adolescentes son diagnosticados con cáncer cada año; de ellos, el $80 \%$ viven en países de ingresos medios y bajos representando el $90 \%$ de las muertes por esta causa, debido a una presentación tardía de la enfermedad. Esto se explica por la falta de diagnóstico oportuno, las altas tasas de abandono del tratamiento, alta prevalencia de desnutrición y otras comorbilidades, el escaso apoyo paliativo y el acceso limitado a las terapias curativas ${ }^{2}$.

En Colombia, las estadísticas muestran que entre el año 2005-2013 dentro de las grandes causas de mortalidad en menores de 18 años, las neoplasias representaron una tasa específica promedio del $5 \%$ para ambos sexos, siendo las leucemias el reporte mayor de muerte en niños (48.3\%) y niñas (47.3\%). El número de menores atendidos en los servicios de salud por diagnóstico de cáncer en el año 2014, fue de 19.834 menores, según datos del Ministerio de Salud y Protección Social3.

En el proceso evolutivo del cáncer, tanto el niño(a) que lo padecen, como sus familiares y allegados, deben aprender vivir con los cambios multidimensionales (físicos, sociales, psicológicos y espirituales) generados por esta condición que afecta su bienestar ${ }^{4}$.

Desde el momento en el que se conoce el diagnóstico y pronostico, tanto el niño(a) como su familia, inician un proceso de adaptación que implica la comprensión de la enfermedad, el control de los síntomas, el cumplimiento del tratamiento y los cambios en el estilo de vida, en los que se ven involucradas medidas de afrontamiento como, la búsqueda de información, búsqueda de apoyo social, expresiones emocionales abiertas, alteraciones en la espiritualidad, mecanismos de focalización, evitación y/o reevaluación, que son manifiestos en mayor o en menor grado, dependiendo de las características propias del individuo $0^{5,6}$.

Con los hallazgos que actualmente se tienen sobre el afrontamiento y la adaptación en cuidadores de personas con enfermedad crónica, se ha generado un importante interés desde el cuidado de enfermería, por comprender las respuestas humanas que se originan en las vivencias de afrontamiento que presentan estas personas en el proceso de su enfermedad 7,8 . Para el caso específico del cáncer en niños(as), se han estudiado diferentes fenómenos que afectan al cuidador de los niños(as); en los cuales se precisa que, teniendo en cuenta que la primera infancia en sí misma requiere un cuidado especial y personalizado, mayor aun, es la exigencia que genera el hecho de ayudar al crecimiento y desarrollo de un niño(a) con cáncer, evidenciado un mayor nivel de incertidumbre, sobre carga de cuidado y alteración de la calidad de vida en padres y cuidadores principales ${ }^{9-11}$.

Además, se han realizado estudios que describen los efectos tardíos que a largo plazo se han producido en el afrontamiento de las familias de niños(as) sobrevivientes de cáncer durante varios años en los que experimentaron el tratamiento del niño(a), o años después del mismo. De estas 
investigaciones, los resultados cuantitativos describen fenómenos como: la presencia de síntomas psiquiátricos y malestar psicológico, dificultades en el funcionamiento familiar, preocupaciones y tensiones del cuidador, presencia de estrés postraumático, presencia de ansiedad y necesidad de ajuste marital ${ }^{12}$. Así mismo, los estudios cualitativos han descrito temas relacionados con el funcionamiento familiar, el ajuste marital, experiencias que generaron un cambio positivo y la preocupación que produce el proceso de la enfermedad ${ }^{13}$.

Con respecto al proceso de afrontamiento y adaptación, Callista Roy plantea que las personas como sistemas adaptativos multidimensionales, se encuentran en continua interacción con un ambiente cambiante ${ }^{14}$. De esta forma el nivel de adaptación se clasifica en aquellos procesos de vida que se encuentran en un nivel integrado, compensatorio o comprometido que cambian constantemente según la presencia y el efecto combinado de tres tipos de estímulos: focales, contextuales y residuales.

Para lograr una adaptación a los diferentes procesos de vida, la persona genera mecanismos de afrontamiento innatos o adquiridos que le permiten actuar ante los cambios producidos en su entorno; de esta forma, se generan respuestas de adaptación que fomentan la integridad de la persona en función de sus necesidades e intereses $^{15}$. Roy plantea que el papel de la enfermera es promover las respuestas de adaptación en modalidades como: el aspecto fisiológico-físico, el autoconcepto-identidad grupal, el desempeño de funciones y la interdependencia ${ }^{16}$. Esto requiere que enfermería reconozca las necesidades de cuidado para una intervención adecuada e individualizada, teniendo en cuenta que cada persona reacciona y manifiesta sus mecanismos de afrontamiento con diferentes respuestas humanas, según como se generen los estímulos.

Dadas las estadísticas de morbilidad y mortalidad relacionadas con el cáncer, el contexto demográfico en el que se propicia y los diferentes estímulos que se producen en el proceso de la enfermedad, se genera la necesidad de mejorar los cuidados integrales especializados dirigidos no solo al niño(a), sino también a sus cuidadores principales, para garantizar una mayor supervivencia. En este sentido, el papel de enfermería reviste una gran responsabilidad ya que está demostrado que en los países de ingresos medios y bajos, la falta de cuidado especializado por parte de enfermería compromete los procesos educativos, asistenciales y de acompañamiento que deben recibir los niños(as) y sus familiares en la enfermedad ${ }^{2}$.

Por estos motivos, el acompañamiento que se realiza desde el cuidado de enfermería es de vital importancia, debido a que, a pesar de los avances tecnológicos para el control de la enfermedad, la palabra cáncer en sí misma, causa temor e intimidación, provocando reacciones de angustia que se asocian con dolor y muerte, que genera grandes problemas en el proceso de adaptación de la enfermedad, cuando no son abordadas por un cuidador especializado.

Para la disciplina de enfermería el modelo de Roy ofrece una descripción amplia de la forma en que enfermería debe centrar el cuidado en los procesos humanos vitales permitiendo establecer sus formas hacia la potencialización de los mecanismos de afrontamiento y de adaptación, así como el conocimiento de las problemáticas biopsicosociales de cada sujeto, desarrollando estrategias encaminadas al mejoramiento de la calidad de vida de las personas ${ }^{17}$.

En tal razón, el presente estudio tuvo como objetivo identificar la capacidad de afrontamiento y adaptación de los cuidadores principales de los niños(as) con cáncer del servicio de Oncólogos de Occidente en la ciudad de Manizales, teniendo como referente investigativo el modelo de adaptación de Callixta Roy.

\section{Materiales y métodos}

Tipo de estudio y diseño. Estudio con enfoque cuantitativo de tipo descriptivo y de corte transversal realizado en el servicio de Oncólogos de Occidente, sede Hospital Infantil de Manizales, Colombia. 
Población y muestra. La población la conformaron los cuidadores principales de niños(as) con cáncer que se encontraban hospitalizados o asistían a quimioterapia ambulatoria, de los cuales se obtuvo una muestra no probabilística de 23 participantes quienes constituyen el total de las personas que decidieron participar en el estudio $y$ que cumplieron con los siguientes criterios de inclusión: personas mayores de 18 años, ser el cuidador principal del niño(a) y llevar mínimo dos meses al cuidado. Se excluyeron los cuidadores de pacientes terminales o con enfermedad en fase de descompensación.

Técnicas e instrumentos de recolección de información. Para identificar el nivel de afrontamiento de los participantes se utilizó la Escala de Medición del Proceso de Afrontamiento y Adaptación versión en español (EsCAP abreviada), validada en la población colombiana, previa autorización para ser aplicada en el presente estudio.

La escala original fue diseñada por Callista Roy en el 2004 y validada a la versión española en Colombia el año 2006, reportando un alfa Cronbach de 0,88 y una correlación Spearman $(r=0,94)$ confirmando su validez facial y confiabilidad $^{18}$. La versión abreviada de la escala fue validada en la población colombiana en el año 2013 , reportando una validez facial aparente del 95\%, un índice de validez de contenido según propuesta de Lawshe de 0,83 y una consistencia interna con un alfa de Cronbach de $0,71^{19}$.

La escala abreviada utilizada en el presente estudio, está compuesta por 33 ítems en formato Likert con cuatro opciones de respuesta (nunca, casi nunca, casi siempre y siempre). Cada ítem hace referencia a la manera como la persona afronta la situaciones actuales, su relación con experiencias pasadas y lo que visualiza en su futuro. El puntaje global de la escala permite una calificación mínima de 33 y máxima de 99 puntos.

Así mismo, se puede interpretar el nivel de afrontamiento de la siguiente forma: afrontamiento bajo: 33-57 puntos, afrontamiento medio: 58-68 puntos y afrontamiento alto: 69-99 puntos. Por otro lado, los ítems pueden se agrupados en cinco factores: recursivo $\mathrm{y}$ centrado, reacciones físicas y enfocadas, proceso de alerta, procesamiento sistemático, conocer y relacionar, ${ }_{18}$ lo que permite analizar de manera particular los comportamiento, reacciones y estrategias que se presentan en el proceso del afrontamiento y adaptación.

Procesamiento y análisis de la información. Para la operacionalización de la información se creó inicialmente una matriz en Microsoft Excel con posterior procesamiento en el programa estadístico para las Ciencias Sociales (IBM SPSS v. 22). Para el análisis se tuvo en cuenta las variables sociodemográficas como: edad, sexo, escolaridad, estado civil, religión, tiempo a cargo del cuidado y la variable relacionada con el nivel de afrontamiento.

Las variables sociodemográficas se explicaron a través de estadística descriptiva mediante frecuencias, medidas de tendencia central y tablas de contingencia; el afrontamiento fue interpretado mediante puntaje global y por niveles, acorde a la explicación detallada en la escala. Además, se realiza análisis por factores presentando los ítems que obtuvieron mayor frecuencia de respuestas en cada factor del afrontamiento.

Ética. La presente investigación se cataloga como una investigación con riesgo mínimo según Resolución 008430 de 1993, en la que no se realizó ninguna intervención o modificación intencionada de las variables biológicas, fisiológicas, sicológicas o sociales de las personas que participaron en el estudio.

Para la recolección de la información se obtuvo inicialmente la autorización de la institución y el consentimiento informado de cada uno de los participantes a quienes se les explicó el objetivo del estudio y se les animó a participar de manera libre y voluntaria garantizando la confidencialidad y el cumplimiento de los principios de autonomía, beneficencia y no maleficencia. 


\section{Resultados}

\section{Características sociodemográficas}

Los 23 cuidadores participantes pertenecen al sexo femenino, dentro de las cuales se encuentran madres y abuelas, cuyas edades oscilan entre $18 \mathrm{y}$ 70 años, con una media de 39 años. El estrato socioeconómico que prevalece es el 2, representando el $43,5 \%$ de la muestra, seguido del estrato 1 con un 30,4\%. En cuanto al nivel de escolaridad, se encuentra mayor representación de mujeres cuidadoras con estudios de bachillerato incompleto en un $34,8 \%$, seguido de bachillerato completo con el $21,7 \%$.

Con respecto al estado civil, se encontró una proporción igual de mujeres solteras y casadas, representando cada grupo un 30,4\%; el resto de la muestra vive en unión libre $26,1 \%$ y se encuentran separadas el 13\%. La mayoría de las cuidadoras profesan la religión católica con representación de un 73,9\%, seguido de la religión cristiana con un 17,4\%. El tiempo mínimo de cuidado reportado por ellas es de 3 meses y el máximo de 10 años, con un promedio de 1,7 años de estar al cuidado de los niños(as).

\section{Nivel de afrontamiento}

En el puntaje global de la escala se obtuvo un promedio de 71 puntos, lo que ubica a las cuidadoras en un afrontamiento alto. El dato mínimo reportado en la escala fue de 64 puntos y el máximo de 81 puntos. Con respecto al nivel de afrontamiento, se evidencia que el $43,5 \%$ de las cuidadoras se ubican en un afrontamiento medio y el $56,5 \%$ en afrontamiento alto. Al analizar el nivel de afrontamiento encontrado teniendo en cuenta las variables sociodemográficas, se observa que las cuidadoras que presentan afrontamiento alto se caracterizan por ser adultas maduras, con estado civil soltera o casada, con nivel de escolaridad en bachiller completo, que viven en el estrato 1 y que profesan la religión católica. Con relación al tiempo en el cuidado del niño(a) se evidencia alto nivel de afrontamiento en las cuidadoras que llevan menos de un año a cargo del cuidado. (Tabla 1)

Por último, se realiza un análisis de los ítems agrupados por factores de afrontamiento, reconociendo aquellos que presentaron las mayores frecuencias reportadas en las opciones de respuesta, encontrando que; en cuanto a los comportamientos personales y los recursos para ser creativas y buscar resultados (Factor recursivo centrado), la mayoría de las cuidadoras refieren que cuando tienen un problema lo aceptan e intentan resolverlo buscando nuevas soluciones o realizando cambios drásticos en sus vidas si es necesario; sin embargo, la totalidad de ellas expresan reaccionar frente a estas situaciones de manera exagerada y en su mayoría, nunca o casi nunca son capaces de orientar sus sentimiento de manera provechosa ni desarrollar un plan para resolver su situación.

Con relación a las reacciones físicas para el manejo de las situaciones (Factor físico enfocado) se observa, que ante las situaciones difíciles, existe una mayor tendencia a no presentar confusión o a bloquearse frente a estos problemas; sin embargo, se experimentan mecanismos de somatización frente a ellos y en la mayoría de las cuidadoras casi siempre o siempre resulta difícil hablar sobre el problema. En cuanto a los comportamientos que se generan para poder procesar la información frente a los problemas (Factor proceso de alerta), se puede apreciar que la mayoría de las cuidadoras, presentan la capacidad para estar atentas y actuar frente a situaciones difíciles adoptando nuevas habilidades para resolver la situación en caso de requerirlo; a pesar de esto, la mayoría de ellas siente que casi nunca se han desempeñado bien manejando problemas complicados.

Así mismo, frente a las estrategias físicas con que cuentan para hacer frente a las situaciones de una manera metódica (Factor procesamiento sistemático), se observa que las cuidadoras tienden a buscar información y a tomarse el tiempo suficiente para comprender la situación y las posibles soluciones. Por último, en cuanto a las estrategias utilizadas para recurrir a sí mismas y a otros utilizando la memoria y la capacidad de imaginación (Factor conocer y relacionar), se observa una mayor tendencia a buscar todos los medios posibles para atender un problema y ver en el mismo las oportunidades y retos. (Tabla 2) 
Tabla 1. Características sociodemográficas y nivel de afrontamiento de las cuidadoras de niños(as) con cáncer

\begin{tabular}{|c|c|c|c|c|}
\hline \multirow{2}{*}{ Variable } & \multicolumn{4}{|c|}{ Puntaje categorizado } \\
\hline & Categoría & $\begin{array}{l}\text { \% Afrontamiento } \\
\text { medio (58-68) }\end{array}$ & $\begin{array}{c}\% \text { Afrontamiento alto } \\
(69-99)\end{array}$ & \%Total \\
\hline \multirow{4}{*}{ Grupos de edad } & Adulto joven (18-34 años) & 17,4 & 13,0 & 30,4 \\
\hline & Adulto maduro (35-64 años) & 21,7 & 43,5 & 65,2 \\
\hline & Adulto mayor (> 64 años) & 4,3 & 0 & 4,3 \\
\hline & Total & 43,5 & 56,5 & 100,0 \\
\hline \multirow{5}{*}{ Estado civil } & Unión libre & 13,0 & 13,0 & 26,1 \\
\hline & Separada & 4,3 & 8,7 & 13,0 \\
\hline & Casada & 13,0 & 17,4 & 30,4 \\
\hline & Soltera & 13,0 & 17,4 & 30,4 \\
\hline & Total & 43,5 & 56,5 & 100,0 \\
\hline \multirow{8}{*}{ Nivel de escolaridad } & Primaria incompleta & 4,3 & 8,7 & 13,0 \\
\hline & Primaria completa & 4,3 & 4,3 & 8,7 \\
\hline & Bachillerato incompleto & 21,7 & 13,0 & 34,8 \\
\hline & Bachillerato completo & 4,3 & 17,4 & $21, \%$ \\
\hline & Técnico & 4,3 & 4,3 & 8,7 \\
\hline & Tecnólogo & ,0 & 4,3 & 4,3 \\
\hline & Universitario & 4,3 & 4,3 & 8,7 \\
\hline & Total & 43,5 & 56,5 & 100,0 \\
\hline \multirow{4}{*}{ Nivel socioeconómico. } & Estrato 1 & 8,7 & 21,7 & 30,4 \\
\hline & Estrato 2 & 26,1 & 17,4 & 43,5 \\
\hline & Estrato 3 & 8,7 & 17,4 & 26,1 \\
\hline & Total & 43,5 & 56,5 & 100,0 \\
\hline \multirow{5}{*}{ Religión } & Católico & 30,4 & 43,5 & 73,9 \\
\hline & Cristiana & 8,7 & 8,7 & 17,4 \\
\hline & Pentecostal & 4,3 & 0 & 4,3 \\
\hline & Ninguna & ,0 & 4,3 & 4,3 \\
\hline & Total & 43,5 & 56,5 & 100,0 \\
\hline \multirow{6}{*}{ Tiempo en el cuidado del niño(a) } & $<1$ año & 13,0 & 34,8 & 47,8 \\
\hline & 1 año & 8,7 & 13,0 & 21,7 \\
\hline & 2 años & 13,0 & 4,3 & 17,4 \\
\hline & 3 años & 4,3 & 4,3 & 8,7 \\
\hline & > 5 años & 4,3 & ,0 & 4,3 \\
\hline & Total & 43,5 & 56,5 & 100,0 \\
\hline
\end{tabular}

Tabla 2. Factores de afrontamiento e cuidadoras de niños con cáncer

\begin{tabular}{|c|c|c|c|c|c|}
\hline \multirow[t]{2}{*}{ Factor } & \multirow[t]{2}{*}{ Ítem } & \multicolumn{4}{|c|}{ Opción respuesta } \\
\hline & & $\begin{array}{l}\text { Nunca } \\
\%\end{array}$ & $\begin{array}{c}\text { Casi } \\
\text { nunca } \\
\%\end{array}$ & $\begin{array}{l}\text { Casi } \\
\text { siempre } \\
\quad \%\end{array}$ & $\begin{array}{l}\text { Siempre } \\
\%\end{array}$ \\
\hline \multirow{6}{*}{ 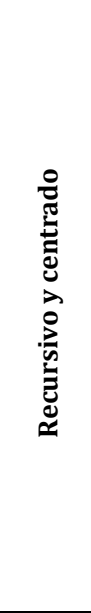 } & $\begin{array}{l}\text { Cuando tengo un problema o situación difícil, acepto el } \\
\text { problema como es o como se presenta }\end{array}$ & 0 & 0 & 17,4 & 82,6 \\
\hline & $\begin{array}{l}\text { Con el fin de salir de un problema o situación difícil puedo } \\
\text { hacer cambios drásticos en mi vida }\end{array}$ & 8,7 & 4,3 & 4,3 & 82,6 \\
\hline & $\begin{array}{l}\text { Cuando tengo un problema o situación difícil, propongo } \\
\text { diferentes soluciones para resolver la situación aunque estas } \\
\text { parezcan difíciles de alcanzar }\end{array}$ & 0 & 8,7 & 8,7 & 82,6 \\
\hline & $\begin{array}{l}\text { Tiendo a reaccionar de manera exagerada cuando tengo un } \\
\text { problema o situación difícil }\end{array}$ & 0 & 0 & 0 & 100 \\
\hline & $\begin{array}{l}\text { Cuando tengo un problema o situación difícil, intento orientar } \\
\text { mis sentimientos y emociones para enfocarlos de forma } \\
\text { provechosa }\end{array}$ & 43,5 & 17,4 & 30,4 & 8,2 \\
\hline & $\begin{array}{l}\text { Cuando tengo un problema o situación difícil, desarrollo un } \\
\text { plan que tiene una serie de acciones o pasos para enfrentar la } \\
\text { situación }\end{array}$ & 47,8 & 13,0 & 30,4 & 8,7 \\
\hline
\end{tabular}




\begin{tabular}{|c|c|c|c|c|c|}
\hline$\frac{8}{\pi}$ & $\begin{array}{l}\text { Cuando tengo un problema o situación difícil, tiendo a } \\
\text { bloquearme y confundirme al menos por un tiempo }\end{array}$ & 65,2 & 17,4 & 13 & 4,3 \\
\hline $\overrightarrow{0}$ & $\begin{array}{l}\text { Cuando tengo un problema o situación difícil, experimento } \\
\text { cambios en el funcionamiento de mi cuerpo }\end{array}$ & 0 & 4,3 & 13 & 82,6 \\
\hline$\overline{\underline{n}}$ & $\begin{array}{l}\text { Cuando tengo un problema o situación difícil, siento que es } \\
\text { complicado hablar del problema }\end{array}$ & 39,1 & 8,7 & 39,1 & 8,7 \\
\hline \multirow{4}{*}{ 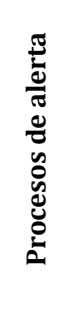 } & $\begin{array}{l}\text { Cuando tengo un problema o situación difícil adopto } \\
\text { rápidamente nuevas habilidades para resolverlo }\end{array}$ & 0 & 4,3 & 4,3 & 91,3 \\
\hline & $\begin{array}{l}\text { Mi capacidad para actuar mejora cuando me encuentro en } \\
\text { situaciones estresantes }\end{array}$ & 0 & 8,7 & 8,7 & 82,6 \\
\hline & $\begin{array}{l}\text { Cuando tengo un problema o situación difícil, me siento } \\
\text { atento y activo durante el día }\end{array}$ & 0 & 0 & 30,4 & 69,6 \\
\hline & Me desempeño bien manejando problemas complicados & 0 & 65,2 & 8,7 & 26,1 \\
\hline \multirow{2}{*}{ 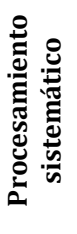 } & $\begin{array}{l}\text { Cuando tengo un problema o situación difícil, intento aclarar } \\
\text { cualquier tipo de duda relacionada con la situación antes de } \\
\text { actuar }\end{array}$ & 0 & 0 & 13,0 & 87,0 \\
\hline & $\begin{array}{l}\text { Cuando tengo un problema o situación difícil, me tomo el } \\
\text { tiempo necesario para comprender la situación y sus posibles } \\
\text { soluciones. }\end{array}$ & 0 & 4,3 & 13,0 & 82,6 \\
\hline \multirow{2}{*}{ 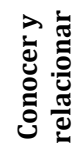 } & $\begin{array}{l}\text { Cuando tengo un problema o situación difícil, busco todos los } \\
\text { medios posibles para enfrentar la situación. }\end{array}$ & 0 & & 4,3 & 91,3 \\
\hline & $\begin{array}{l}\text { Veo las situaciones difíciles o problemas como una } \\
\text { oportunidad o un reto. }\end{array}$ & 17,4 & 4,3 & 8,7 & 69,6 \\
\hline
\end{tabular}

\section{Discusión}

Desde el inicio de una enfermedad crónica, los niños(as) dependen de los cuidados que proveen los adultos. Cuando se elige el cuidador principal o primario, es generalmente la madre quien asume este rol, ya que culturalmente se considera como la persona idónea para facilitar los cuidados $^{20}$. En el presente estudio, el $100 \%$ de las participantes eran madres y abuelas quienes asumieron el rol de cuidadores principales desde

el momento en que los niños(as) fueron diagnosticados con cáncer. Estos resultados han sido reportados en otros estudios donde se ha descrito de la misma forma, que la mayor parte de los cuidadores principales de niños(as) con cáncer son mujeres ${ }^{6-9,21,22}$.

Por otro lado, al analizar al nivel de afrontamiento y las variables sociodemográficas de las participantes, se puede decir que a pesar de que la mayor representatividad de las cuidadoras se concentra en el estrato 2 y poseen un nivel de escolaridad relacionado con el bachiller incompleto, las que demuestran un mayor afrontamiento son aquellas que pertenecen al estrato 1 y que poseen bachillerato completo.

Es innegable que en el proceso de la enfermedad, se genera un contexto para el niño(a) y sus cuidadores en el que experimentan una serie de sentimientos en torno a la noticia del diagnóstico, que va de la mano con una amplia lista de deberes que se deben empezar a cumplir dando paso a un proceso que involucra: exámenes, procedimientos invasivos, suministro de medicamentos citotóxicos, cambio de hábitos para el niño(a) en su alimentación, abandono de la escuela y procesos de aislamiento protector, entre otros.

Así mismo, para el cuidador principal del niño(a) se puede generar la necesidad del abandono súbito de su rol primario y secundario en el hogar, incluso del rol laboral, los cuales se constituyen en entradas o estímulos focales y contextuales que 
permiten la activación de subsistemas de afrontamiento que deben manejarse efectivamente para lograr una adaptación a este nuevo fenómeno que se ha instaurado en sus vidas.

En este sentido, y basados en el modelo de afrontamiento y adaptación de Roy, las cuidadoras de los niños(as) deben activar sistemas reguladores de afrontamiento que van desde la activación de mecanismos reguladores de tipo neuronal, químico y endocrino, hasta las reacciones y comportamientos que se asumen para percibir, procesar la información, aprender, adoptar un juicio personal y emociones que permitan adaptarse a esa nueva situación ${ }^{15}$. En este caso, las cuidadoras deberán alcanzar principalmente dos modos de adaptación citados por Roy que se relacionan con la adaptación de los aspectos psicosociales y espirituales, y con la adaptación en la función del rol social, que podrían encontrarse afectados por las vivencias de afrontamiento demostradas por ellas, en las que se privilegia un estado de alerta permanente y hacer frente a los problemas, independiente de los cambios que se requieran generar en el estilo de vida.

Si bien los resultados del presente estudio evidencian un nivel de afrontamiento medio y alto, demostrado a través de reacciones, comportamientos y estrategias utilizadas para hacer frente a los problemas difíciles, es importante tener en cuenta, que en su totalidad las cuidadoras refieren un mecanismo exagerado para reaccionar frente a estos problemas y mecanismos de somatización en la mayoría de los casos; hecho que demuestra la presencia de estrés asumido en el proceso del cuidado.

Estos hallazgos, coinciden con el estudio realizado por Margarita Chacin, et al. ${ }^{5}$, quienes al indagar sobre las estrategias de afrontamiento en padres de niños y adolescentes con cáncer, encontraron que la mayoría de los padres afrontan la enfermedad de sus hijos enfocándose en el problema, es decir, se dirigen a la definición del problema como tal buscando soluciones alternativas sobre la base de su costo y beneficio, y de su elección y aplicación; además, identificaron que los padres utilizan menor grado de estrategias basadas en la emoción, con lo que consiguen disminuir el trastorno emocional, mostrarse a la defensiva y actuando sobre las respuestas emocionales y físicas relacionadas con el estrés, para poder mantener el ánimo, y continuar funcionando bien.

De igual forma, un estudio cualitativo realizado por Espada et al.,22 en el que se realizaron sesiones grupales de entrevista a padres de niños con cáncer, describe diversos temas encontrados con relación a las estrategias de afrontamiento utilizadas por los padres, entre las cuales se mencionan las estrategias centradas en el problema y la búsqueda de información de los padres; datos que coinciden con los hallazgos del presente estudio.

Por otro lado, se observa un hallazgo a tener en cuenta, que se relaciona con el hecho de que las cuidadoras a pesar de demostrar en sus mecanismos de afrontamiento, disposición bajo sistemas cognitivos y procesos activados de alerta para asumir las situaciones en el cuidado del niño(a), revelan dificultades para comunicar dichos problemas y muchas de ellas, tienen la percepción de casi nunca haberse desempeñado bien en el manejo de los problemas complicados. Un estudio previo donde se tomó como referencia el modelo de adaptación de Roy en padres de niños en posoperatorio de cardiopatía congénita, demuestra cómo enfermería puede fortalecer a través del cuidado, la comunicación de experiencias, la expresión y la activación de redes de apoyo secundarias para que el proceso de afrontamiento promuevan la regulación de la emoción y estrategias dirigidas a la solución del problema, que marca la diferencia en el proceso de afrontamiento entre padres solos y acompañados 23 .

Los hallazgos demuestran la importancia del acompañamiento que enfermería realice a este tipo de cuidadoras, en donde se incorpore la promoción de la salud mental, teniendo en cuenta que la mayoría de las cuidadoras con presentación de afrontamiento alto, son las que llevan menos de un año en el rol de cuidadores principales, por tal motivo, se considera 
pertinente prevenir la carga de cuidado que se llegue a generar en el transcurso del tiempo, y con ella todas las consecuencias que se derivan por el hecho de hacer frente a los problemas mediante mecanismos constantes de afrontamiento activo donde posiblemente se agoten los sistemas de alerta y las estrategias utilizadas para afrontar las situaciones en el caso de no ser para ellas satisfactorios los resultados.

En conclusión en el estudio se encontró un nivel de afrontamiento alto en más de la mitad de las cuidadoras que eran adultas maduras, pertenecientes al estrato 1 , con nivel de escolaridad de bachiller incompleto y que llevaban menos de un año al cuidado del niño(a). Con respecto al estado civil se evidenció este mismo nivel de afrontamiento tanto para las cuidadoras solteras como casadas. Por otro lado, se debe considerar que casi la mitad de la muestra presentó un afrontamiento medio, siendo observado en cuidadoras con similares características sociodemográficas a las que presentaron afrontamiento alto, en cuanto a la edad; sin embargo, en este grupo se encontraba un porcentaje representativo de mujeres que vivían en unión libre y con igual porcentaje de cuidadores que llevaban menos de un año y dos años al cuidado del niño(a).

Es importante realizar otros estudios que demuestren relaciones estadísticamente significativas entre el nivel de afrontamiento y las características de los cuidadores, con el fin de posibilitar el diseño de estrategias y planes de acompañamiento por parte de enfermería, teniendo en cuenta las características que definen al cuidador del niño(a) y que podrían potenciar un afrontamiento integrado.

Conflicto de intereses: Ninguno declarado por autores.

\section{Referencias}

1. República de Colombia. Ministerio de Salud y Protección Social - Colciencias. Guía de práctica clínica para la detección oportuna, diagnóstico y seguimiento de leucemia linfoide aguda y leucemia mieloide aguda en niños, niñas y adolescentes. Guía No. 9. 2013:40-44.
2. Rodríguez-Galindo C, Friedricha P, Morrisseya L. Frazier L. Global challenges in pediatric oncology. Curr Opin Pediatr. 2013;25(1):3-15.

3. República de Colombia. Ministerio de Salud y Protección Social. Dirección de Epidemiología y Demografía. Situación del cáncer en niños, niñas, adolescentes y jóvenes en Colombia. Bogotá; 2015.

4. Flórez-Torres IE, Montalvo-Prieto A, Herrera-Lían A, Romero-Massa E. Afectación de los bienestares en cuidadores de niños y adultos con enfermedad crónica. Rev. salud pública. 2010;12(5):754-764.

5. Chacín-Fuenmayor M, Chacín J. Estrategias de afrontamiento en padres de niños y adolescentes con cáncer. Rev Venez Oncol. 2011;23(3):199-208.

6. Dos Santos-Alves DF, Guirardello EdeB, YamaguchiKurashima A. Estrés relacionado al cuidado: el impacto del cáncer infantil en la vida de los padres. Rev. LatinoAm. Enfermagem. 2013;21(1):[07 pantallas].

7. Grau C, Fernández Hawrylak M. Familia y enfermedad crónica pediátrica. Anales Sis San Navarra. 2010;33(2): 203-212.

8. Pinto-Afanador N. Bienestar espiritual de los cuidadores familiares de niños que viven enfermedad crónica. Investigación en Enfermería: Imagen y Desarrollo. 2011;9(1):19-35.

9. Rubira-do-Espírito-Santo EA, Munhoz-Gaíva MA, Martínez Espinosa M, Barbosa DA, Silva-Belasco AG. Cuidando del niño con cáncer evaluación de la sobrecarga y calidad de vida de los cuidadores. Rev. Latino-Am. Enfermagem. 2011;19(3).

10. Ballestas-Cueto H, López-Ortega E. Meza-Bustillo R, Palencia-Suárez K, Ramos-Polo D. Montalvo-Prieto A. Cuidadores familiares de niños con cáncer y su funcionalidad. Salud Uninorte. 2013;29(2):249-259.

11. Barrera-Ortiz L, Sánchez-Herrera B, Carrillo González GM. La carga del cuidado en cuidadores de niños con enfermedad crónica. Revista Cubana de Enfermería 2013;29(1):39-47.

12. Ljungman $L$, Cernvall $M$, Grönqvist $H$, Ljótsson B. Ljungman G, von Essen L. Long-Term positive and negative psychological late effects for parents of childhood cancer survivors: A systematic review. PLoS ONE. 2014;9(7):e103340.

13. Grau-Rubio C, Espada-Barón MC. Percepciones de los padres de niños enfermos de cáncer sobre los cambios en las relaciones familiares. Psicooncologia. 2012; 9 (1): 125-136.

14. Carmen Gutiérrez MC. Adaptación y cuidado en el ser humano. Bogotá. Universidad de la Sabana. 2007.

15. Phillips KD, Harris Robín. Modelo de adaptación de Callista Roy. En: Raile-Alligood. Modelos y Teorías de Enfermeria. 8a ed. España. Elsevier; 2015: 293-313.

16. Cutcliffe J, Mckenna H, Hyrkäs K. Modelos de enfermería: aplicación a la práctica. México. Manual Moderno; 2011:105-130.

17. Moreno-Fergusson ME, Alvarado-García AM. Aplicación del modelo de adaptación de Callista Roy en Latinoamérica: revisión de la literatura. Aquichan. 2009;9(1):62-72.

18. Gutiérrez-López C, Veloza-Gómez MM, MorenoFergusson ME, Durán de Villalobos MM, López de Mesa 
C, Crespo 0. Validez y confiabilidad de la versión en español del instrumento "Escala de medición del proceso de afrontamiento y adaptación" de Callista Roy. Aquichan. 2007;7(1):54-63.

19. Sarmiento-González P, Botero-Giraldo J, CarvajalCarrascal G. Validez de constructo de la Escala de medición del proceso de afrontamiento y adaptación de Roy, versión modificada en español. Index Enferm. 2013;22(4):233-236.

20. Grau C, Fernández-Hawrylack. Familia y enfermedad crónica pediátrica. An. Sist. Sanit. Navar. 2010; 33 (2): 203-212.

21. De la Huerta-H R, Corona-M J, Méndez-V J. Evaluación de los estilos de afrontamiento en cuidadores primarios de niños con cáncer. Revista Neurología, Neurocirugía y Psiquiatría. 2006; 39(1): 46-51.

22. Espada MC, Grau C. Estrategias de afrontamiento en padres de niños con cáncer. Psicooncología. 2012;9(1): 25-40.

23. Suárez-Acuña CE, Monroy-Garzón AM. Afrontamiento y adaptación de los padres durante el postoperatorio de cardiopatía congénita. Index Enferm. 2012;21(3):145149. 